\title{
Actualización del tratamiento de las fracturas intertrocantéricas
}

\author{
Sebastián Pereira, Gabriel Vindver, Fernando Bidolegui \\ Servicio de Ortopedia y Traumatología, Hospital Sirio Libanés, ECICARO, Ciudad Autónoma de Buenos Aires, \\ Argentina
}

\section{RESUMEN}

Las fracturas intertrocantéricas representan el $50 \%$ de todas las fracturas del fémur proximal y su incidencia aumenta debido a la mayor expectativa de vida de la población. La reducción y fijación con un clavo de fémur proximal es el tratamiento de elección. Sin embargo, la falla de la osteosíntesis genera un aumento en la morbilidad y mortalidad, especialmente en el grupo de pacientes más añosos. Numerosos estudios señalan que los principales factores predictivos de falla están relacionados con errores de reducción de la fractura o con una incorrecta colocación del implante. Estos errores pueden ocurrir en distintas etapas de la técnica quirúrgica, como la planificación preoperatoria, la ubicación del paciente, la visualización y la reducción de la fractura, la ubicación del punto de ingreso y la colocación del clavo, y el posicionamiento del elemento (tornillo o lámina) cefálico. Por lo tanto, sobre la base de la bibliografía disponible y las más de 1000 fracturas intertrocantéricas tratadas con clavos de fémur proximal desde abril de 2002 hasta mayo de 2020, nos proponemos describir los posibles errores durante la técnica quirúrgica y ofrecer una guía sistematizada para evitarlos. Conclusiones: A pesar del gran avance y desarrollo de implantes en los últimos años, los principales factores determinantes del resultado final de la fijación de las fracturas intertrocantéricas siguen siendo la calidad de la reducción y el correcto posicionamiento del implante. Conocer los diferentes errores que se pueden producir durante cada uno de los pasos de la técnica quirúrgica resulta indispensable para poder evitarlos.

Palabras clave: Fracturas intertrocantéricas; clavo de fémur proximal, fractura de cadera.

Nivel de Evidencia: $\mathrm{V}$

\section{Treatment Update for Intertrochanteric Fractures}

\section{ABSTRACT}

Intertrochanteric fractures represent $50 \%$ of all proximal femur fractures and their incidence is increasing due to the greater life expectancy of the population. Reduction and fixation with a proximal femoral nail is the treatment of choice. However, the failure of osteosynthesis generates an increase in morbidity and mortality, especially in elderly patients. Numerous studies indicate that the main factors of failure are related to errors in fracture reduction and incorrect implant placement. These errors can occur at different stages of the surgical technique: preoperative planning; patient positioning; visualization and reduction of the fracture; location of the entry point and positioning of the cephalic element (screw or blade). Therefore, based on the existing literature and the experience of more than 1000 intertrochanteric fractures treated with proximal femoral nails from April 2002 to May 2020 , we set to describe possible errors during the surgical technique and provide a systematic guide to avoid them. Conclusion: In spite of the improvements in implant design in recent years, the main factors determining the final outcome of the fixation of intertrochanteric fractures are the quality of reduction and the correct positioning of the implant. Awareness of the different errors that may occur at each stage of the surgical technique is essential to avoid them.

Key words: Intertrochanteric fracture; proximal femoral nail; hip fracture.

Level of Evidence: V 


\section{INTRODUCCIÓN}

Las fracturas intertrocantéricas representan el 50\% de las fracturas del fémur proximal y su incidencia está aumentando debido a la mayor expectativa de vida de la población. ${ }^{1}$ La reducción y fijación con un clavo de fémur proximal es el tratamiento de elección; sin embargo, la falla de la osteosíntesis genera un aumento en la morbilidad y la mortalidad, especialmente en el grupo de pacientes más añosos. ${ }^{2}$ Numerosos estudios señalan que los principales factores predictivos de falla están relacionados con errores de reducción de la fractura o con una incorrecta colocación del implante. ${ }^{3}$ Estos errores pueden ocurrir en distintas etapas de la técnica quirúrgica, como la planificación preoperatoria, la ubicación del paciente, la visualización y la reducción de la fractura, la ubicación del punto de ingreso, la colocación del clavo y el posicionamiento del elemento (tornillo o lámina) cefálico.

Por lo tanto, sobre la base de la bibliografía disponible y de nuestra experiencia personal, nos proponemos realizar una actualización de los conceptos del tratamiento de las fracturas intertrocantéricas con clavos endomedulares, así como describir los posibles errores durante la técnica quirúrgica y ofrecer una guía sistematizada para evitarlos. $^{4}$

\section{PLANIFICACIÓN PREOPERATORIA}

Los aspectos clave que deberán preverse durante la planificación preoperatoria son: el ángulo cervicodiafisario del fémur, el diámetro del canal femoral y la presencia de alguna deformidad que pueda impedir la colocación del implante. Para esto, es indispensable contar con una radiografía de ambas caderas que nos permita evaluar el fémur proximal contralateral sano, y también con una radiografía completa del fémur afectado. En algunas ocasiones, una radiografía del fémur proximal con tracción podrá facilitarnos la interpretación del patrón fracturario. ${ }^{5}$

En la mayoría de los casos, un implante con un ángulo de $130^{\circ}$ será adecuado. Si bien, en los pacientes añosos, por efecto de la pérdida del espesor de las corticales, el canal femoral suele ser mayor que el de los pacientes jóvenes; en ocasiones, puede ser necesario fresar el canal para introducir el clavo sin someter al fémur a fuerzas que puedan generar una fractura iatrogénica.

\section{UBICACIÓN DEL PACIENTE}

La ubicación del paciente en decúbito supino sobre una mesa de tracción es la posición más utilizada. Si bien demanda un tiempo extra y no está exenta de complicaciones perineales, ofrece las ventajas de facilitar una completa visualización del fémur proximal, especialmente en el perfil, y la reducción y mantenerla durante todo el procedimiento. Los puntos clave son: la correcta fijación del pie a la bota de la mesa que nos garantice una tracción efectiva; el cuidadoso acolchado del poste perineal para evitar lesiones del nervio pudendo, de la vulva o escroto ${ }^{6} \mathrm{y}$, por último, el manejo de la extremidad inferior contralateral. Respecto a este último punto, si el paciente presenta un movilidad normal de la cadera contralateral, preferimos abducir la extremidad para facilitar la visualización del fémur proximal en el perfil (Figura 1A). Si la movilidad de la cadera contralateral está limitada, adoptamos la posición en "tijeras" siempre, en ambos casos, ejerciendo tracción en la extremidad para contrarrestar la tracción generada en la pierna afectada y evitar así la báscula de la pelvis (Figura 1B).

Otra alternativa es posicionar al paciente en decúbito supino sobre una mesa común radiolúcida (Figura 1C). La principal ventaja es mantener la extremidad libre permitiendo la movilidad en todos los planos, durante el procedimiento; sin embargo, requiere una tracción manual por un asistente extra y dificulta más la visualización con el arco en "C". 
A
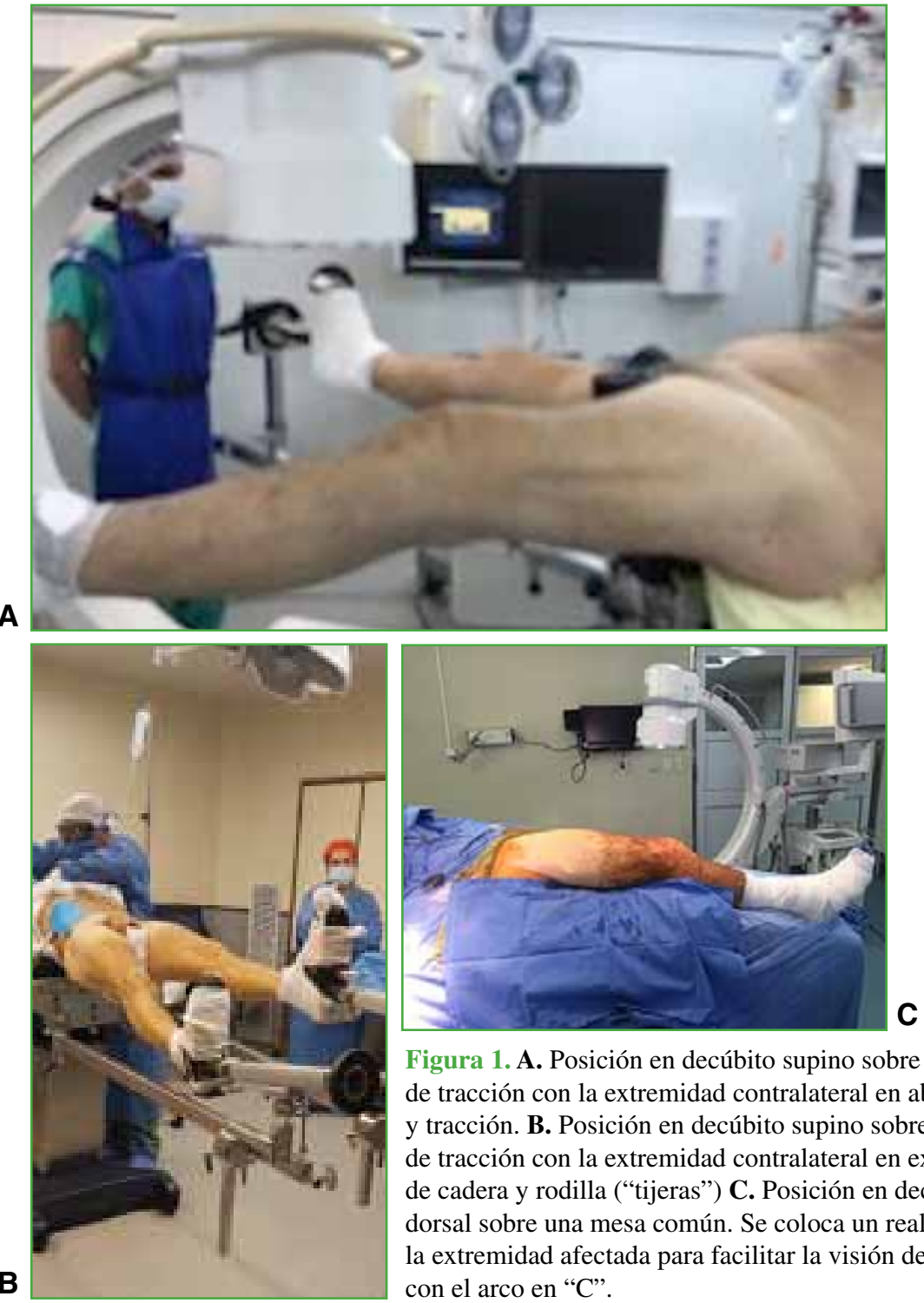

Figura 1. A. Posición en decúbito supino sobre la mesa de tracción con la extremidad contralateral en abducción y tracción. B. Posición en decúbito supino sobre la mesa de tracción con la extremidad contralateral en extensión de cadera y rodilla ("tijeras") C. Posición en decúbito dorsal sobre una mesa común. Se coloca un realce bajo la extremidad afectada para facilitar la visión de perfíl con el arco en "C".

\section{VISUALIZACIÓN DEL FÉMUR PROXIMAL}

La correcta visualización y la óptima reducción de la fractura están íntimamente relacionadas. La probabilidad de una mala reducción o una inadecuada colocación del implante es alta si la visualización no es la correcta. ${ }^{7}$ Las estructuras que se deben identificar claramente tanto en la proyección de frente como en la de perfil son: la cabeza femoral y el espacio articular, el cuello femoral, ambos trocánteres y los dos tercios proximales del fémur. La verdadera proyección de perfil deberá contemplar la anteversión del cuello femoral. Para esto, el rayo del intensificador de imágenes deberá ubicarse entre $0^{\circ}$ y $20^{\circ}$ respecto al plano horizontal hasta lograr trazar una línea recta desde el centro de la cabeza, paralela al cuello femoral y a la diáfisis femoral (Figura 2). ${ }^{7}$ Una vez logradas las imágenes deseadas, resulta útil realizar unas marcas en el piso para señalar el lugar en el que deberá volver a ubicarse el arco en "C" luego de colocar los campos (Figura 3). 

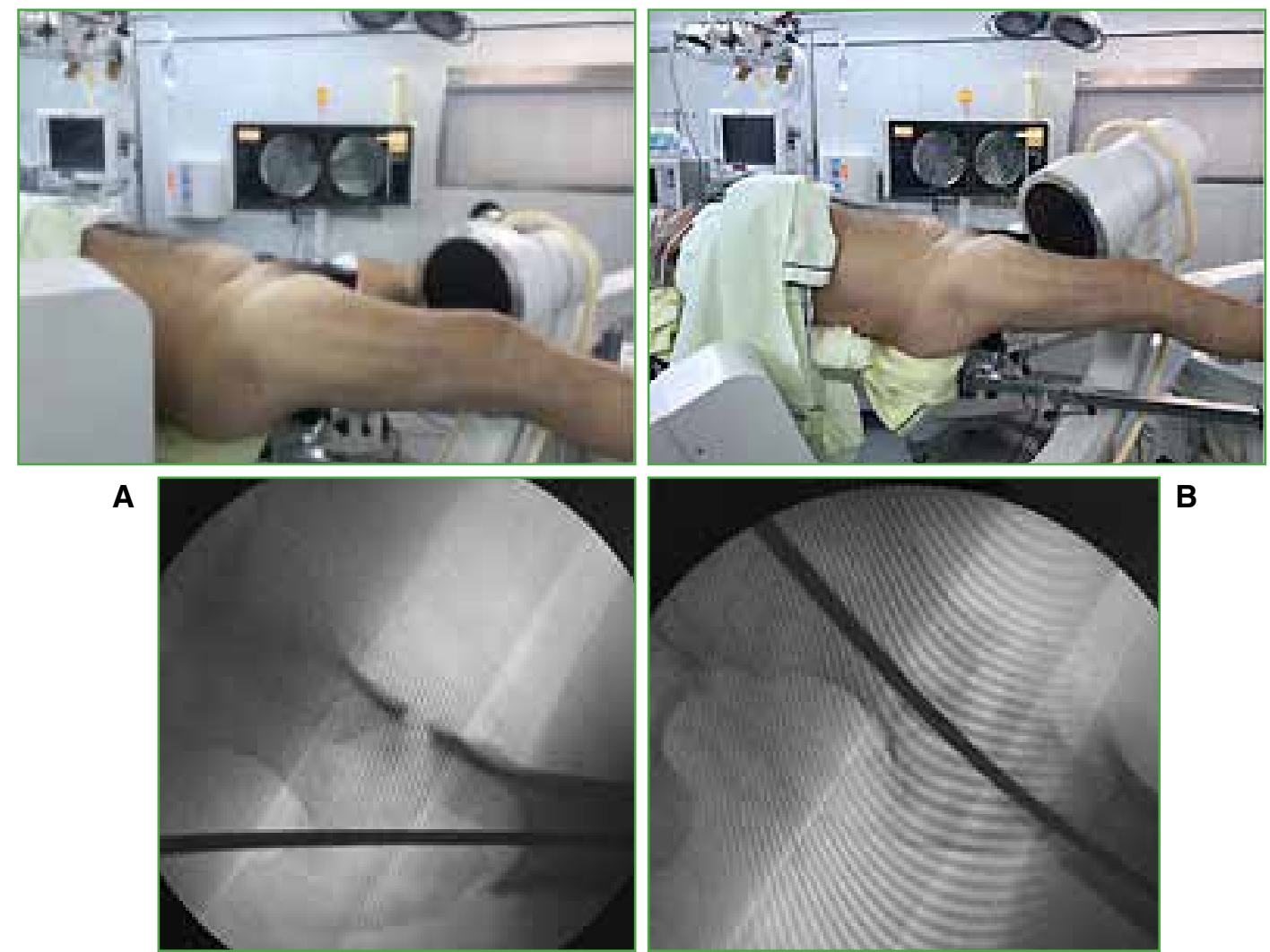

B

Figura 2. A. Imagen de perfil del fémur proximal ( $\operatorname{arco}$ en " $\mathrm{C}$ " a $180^{\circ}$ ) B. Imagen de perfil contemplando la anteversión del cuello femoral (arco en "C" a 160").

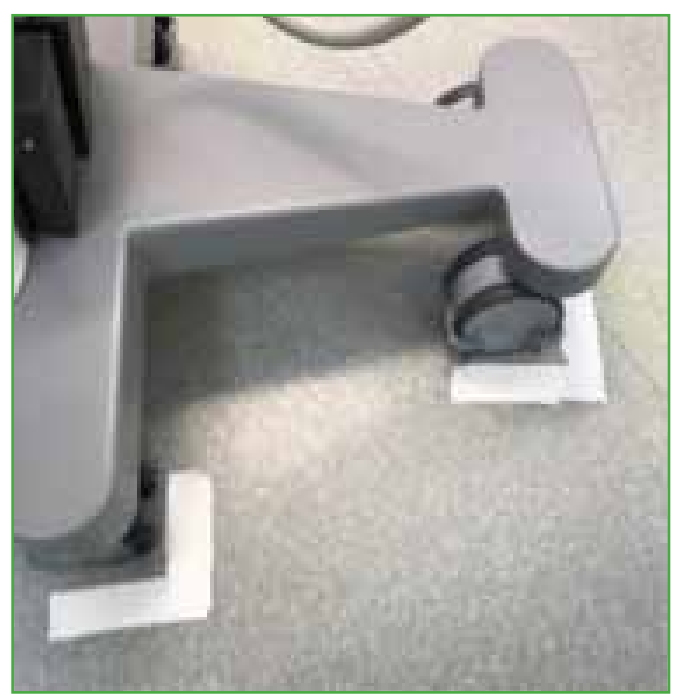

Figura 3. Referencias en el piso para reposicionar el arco en "C". 


\section{REDUCCIÓN DE LA FRACTURA}

En los últimos años, los implantes han sufrido mejoras en el diseño, pero ninguna sustituye una adecuada técnica de reducción. Es notable que, mientras que muchos artículos remarcan la importancia de la calidad de la reducción como principal factor pronóstico de falla, pocos se detienen en describir la técnica de reducción. En 2020, Yoon y cols. comunicaron que, en más del 50\% de las 322 fracturas intertrocantéricas analizadas, la reducción cerrada fue insuficiente y necesitaron gestos de reducción percutánea para lograr una reducción óptima. ${ }^{8}$

\section{a. Reducción en el plano coronal}

Si bien es cierto que la maniobra de tracción y rotación interna, por lo general, resulta en una reducción adecuada; en algunas ocasiones, sobre todo en patrones inestables, puede ser insuficiente. Ottolenghi y cols. alertaron sobre algunas fracturas intertrocantéricas, a las que clasificaron como "extradigitales", porque mantenían la inserción de los músculos rotadores externos, que requieren de una rotación externa del fragmento diafisario para su reducción. ${ }^{9} \mathrm{Si}$, luego de la tracción en el eje y la maniobra de rotación interna o externa, no se logra conseguir el ángulo cervicodiafisario deseado, una leve abducción de la extremidad puede ayudar. Deberá preverse que la posición de la pierna en abducción podrá dificultar la correcta ubicación del punto de ingreso del clavo. Por esta razón, en estas situaciones, lo recomendable es realizar una fijación temporal de la fractura con clavijas para luego poder volver a reposicionar la pierna evitando la pérdida de la reducción.

En la proyección anteroposterior, también deberá evaluarse la continuidad del calcar, ya que, en ocasiones, a pesar de haberse recuperado el ángulo cervicodiafisario puede persistir una traslación de las corticales. En ese caso, el uso de un "clamp colineal" o de un gancho de hueso sobre la cortical medial del cuello o del fragmento distal podrá reducir el desplazamiento.

\section{b. Reducción en el plano sagital}

En ciertos patrones de fractura, el fragmento proximal tiende a flexionarse y la diáfisis, a caer. La primera maniobra para reducir este desplazamiento en el plano sagital suele ser la colocación de un soporte sobre la cara posterior del muslo (puño de la mano, martillo, muleta, etc.) que permita reducir el desplazamiento. Sin embargo, muchas veces, esto suele ser insuficiente. Para estas situaciones, se han descrito algunas maniobras de reducción percutánea. En 2011, Chun y cols. describieron una técnica que consiste en la introducción percutánea de una clavija de Steinmann de 4,2 mm, en la cortical anteromedial del cuello y, ejerciendo fuerza hacia posterior, consiguen reducir la flexión del fragmento proximal (Figura 4). ${ }^{10}$ Yoon y cols. introducen, a través de un pequeño abordaje anterolateral proximal al trocánter menor en el muslo, una pinza hemostática larga y, generando tracción hacia arriba, se corrige el defecto de flexión y rotación del fragmento proximal. ${ }^{11}$

En 2007, Carr describe un patrón de desplazamiento en las fracturas intertrocantéricas conminutas, en las que el fragmento diafisario se acorta y rota externamente, y el fragmento proximal se desplaza en varo y se impacta dentro del fragmento distal generando un solapamiento de las corticales anteriores. Propone, entonces, introducir una clavija de Steinmann entre ambos fragmentos para levantar el fragmento proximal y desimpactarlo del fragmento distal (Figura 5). ${ }^{12}$

\section{UBICACIÓN DEL PUNTO DE INGRESO}

La definición de un punto de ingreso ideal puede ser imposible de establecer, debido a que este depende de las variaciones anatómicas del trocánter mayor y de las variaciones en el ángulo de los distintos tipos de clavos. En un estudio cadavérico utilizando distintos diseños de clavos, Osrtum y cols. definieron la punta del trocánter mayor de 2 a $3 \mathrm{~mm}$ medial a ella como el punto de ingreso "universal" en el plano coronal. También mencionan que se debe evitar el punto de ingreso lateral, porque indefectiblemente genera un desplazamiento en varo del fragmento proximal. $^{13}$

La ubicación del punto de ingreso en el perfil se ha estudiado menos; no obstante, un incorrecto ingreso en este plano puede provocar una fractura iatrogénica del fémur proximal, por lo que se recomienda un punto de ingreso $5 \mathrm{~mm}$ posterior a la punta del trocánter. 


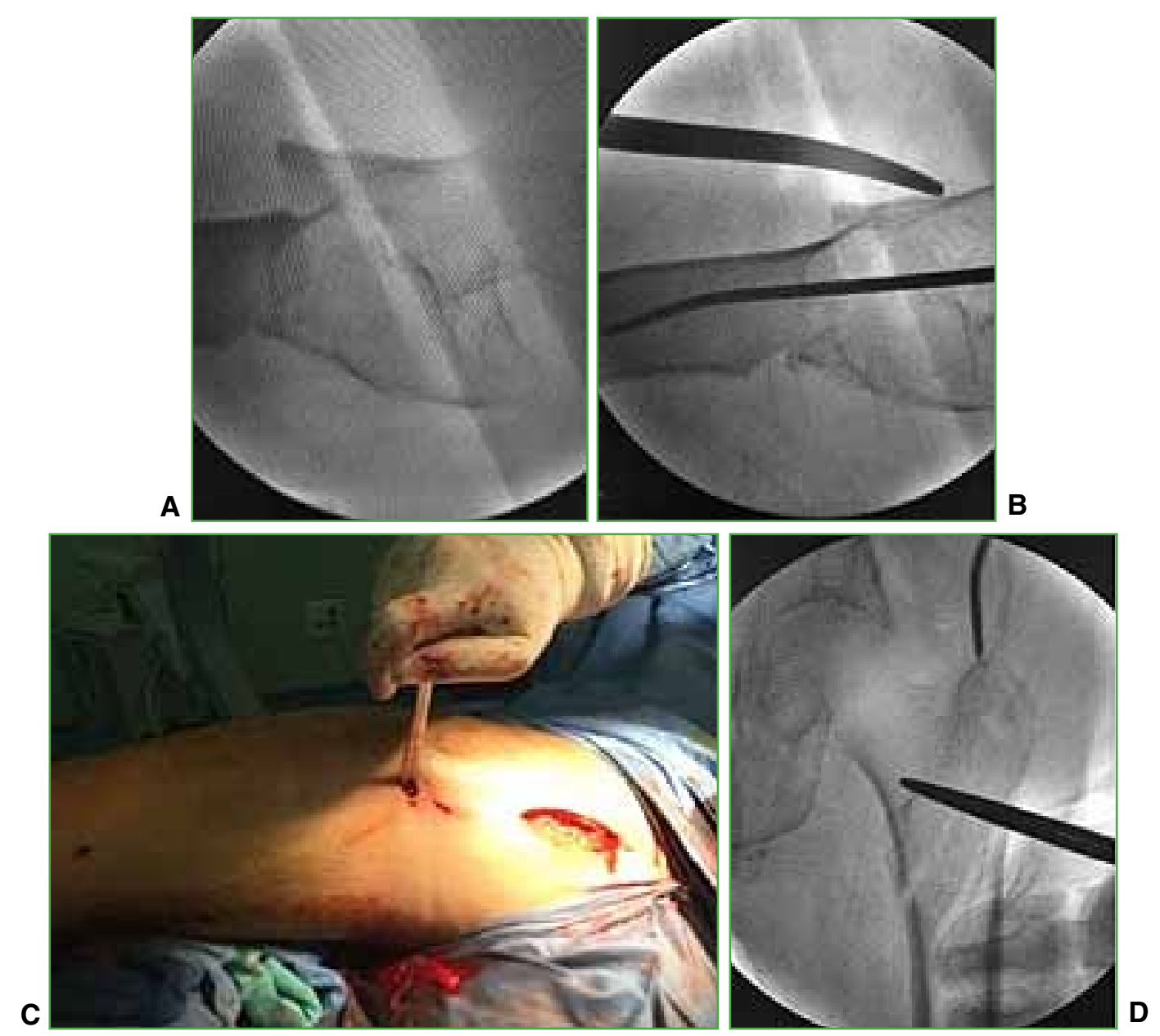

Figura 4. A. Desplazamiento en flexión del fragmento proximal. B y C. Reducción del desplazamiento con una tijera a través de un abordaje percutáneo. D. Imagen radioscópica de la reducción conseguida en el frente.

Una vez definido cuál es el punto de ingreso ideal, se deberán reconocer todos los factores que puedan interferir con su correcto posicionamiento. En este sentido, las situaciones que pueden causar una lateralización del punto de ingreso son: la conminución de la fractura que se extiende hacia la punta del trocánter, la ubicación incorrecta del paciente en la mesa de tracción o mesa de cirugía, la incorrecta preparación de los campos que interfieran con el abordaje o errores en el abordaje. Otro momento en cual inadvertidamente se puede lateralizar el punto de ingreso es en el fresado. No se debe comenzar a fresar hasta tener un contacto íntimo de la fresa con el hueso para evitar un fresado excéntrico y provocar así un desplazamiento lateral del canal de ingreso. ${ }^{13}$

\section{COLOCACIÓN DEL CLAVO}

Una vez fresado el canal de ingreso, procedemos a la introducción del clavo. Habitualmente es posible introducirlo de forma manual, con suaves movimientos de rotación. Solo una vez que el clavo esté alineado con el canal femoral, si es necesario, se podrán aplicar suaves golpes de martillo para alcanzar la posición deseada. Si, a pesar de esto, su descenso no progresa, lo recomendable es retirar el clavo y realizar un fresado del canal para evitar una fractura iatrogénica del fémur.

Luego de introducir el clavo en la posición deseada, sobre todo, si el punto de ingreso fue lateral a la punta del trocánter mayor, se puede generar un desplazamiento secundario en varo del fragmento proximal y lateralización de la diáfisis "efecto cuña". ${ }^{14}$ Es importante detectar esta situación antes de colocar la clavija guía cervicocefálica. 

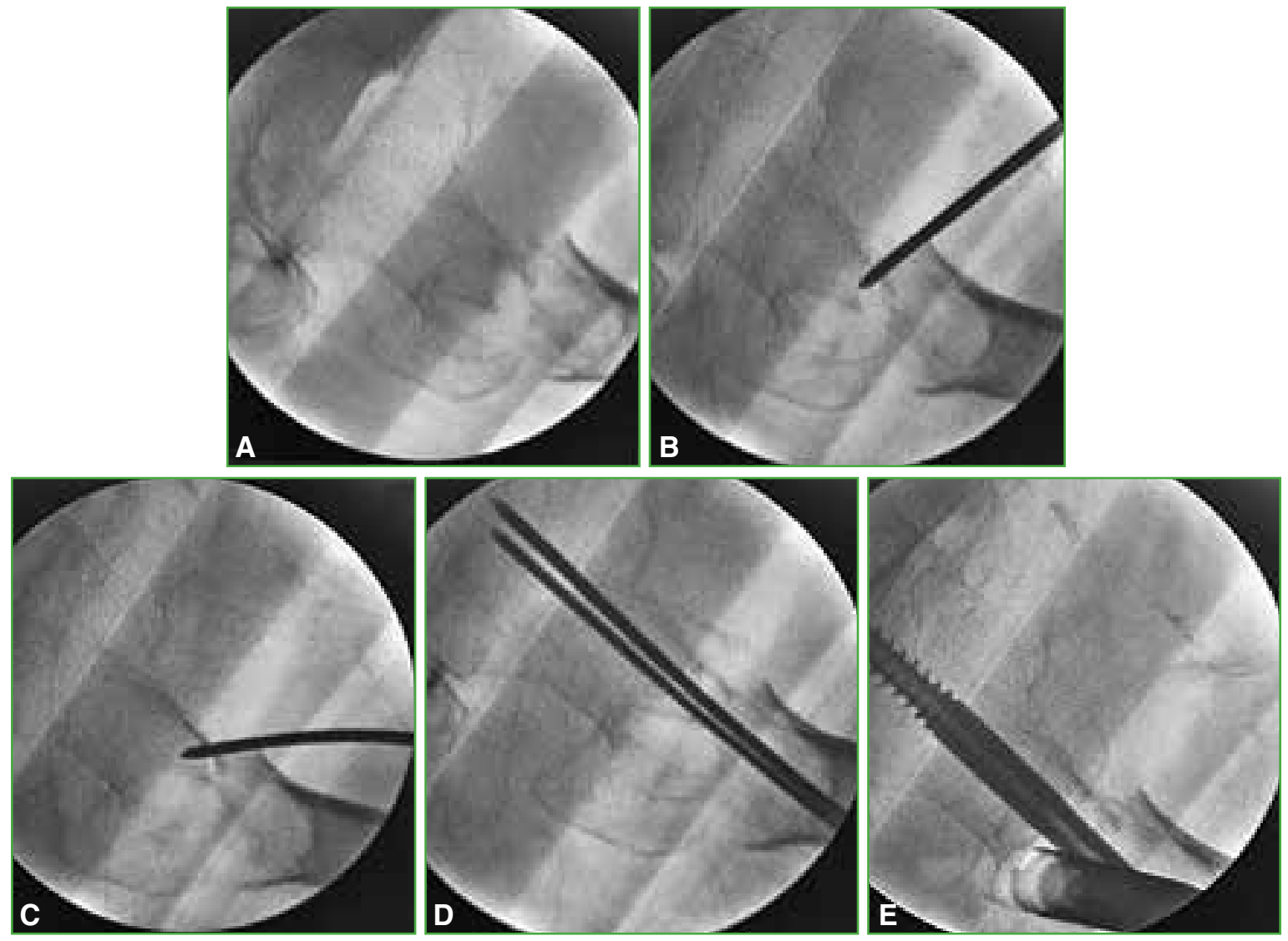

Figura 5. A. Imagen radioscópica de perfil en la que se evidencia la caída posterior del fragmento proximal y el solapamiento de las corticales, lo que imposibilita la reducción cerrada. B y C. Introducción de una clavija de $3 \mathrm{~mm}$ en el foco y reducción del desplazamiento. D. Estabilización transitoria con dos clavijas anteriores procurando que no interfieran con la introducción del clavo. E. Fijación definitiva con el tornillo cefálico.

La solución suele ser aplicar más tracción en el eje o, en algunas circunstancias, aplicar una leve abducción sobre la extremidad. Si, pese a esto, no se consigue la reducción deseada, probablemente el conflicto esté dado por un punto de ingreso lateral. En ese caso, lo recomendable es reposicionar el punto de ingreso. Lo primero que realizamos, luego de extraer el clavo, es comprobar que las partes blandas no estén interfiriendo con el acceso, si esto ocurre, extendemos el abordaje a proximal para permitir un ingreso más alineado con el canal femoral. Si, a pesar de ello, la conminución del trocánter o del canal previamente generado hacen que la clavija se desplace a lateral, volver a fresar el canal de ingreso ejerciendo fuerza hacia medial para remover la cortical superolateral del fragmento cabeza-cuello permitirá darle más lugar al extremo proximal del clavo y, de esta forma, evitar que este provoque un deslazamiento en varo. ${ }^{15}$

Otro desplazamiento posible, aunque poco frecuente, es que, luego de introducir el clavo, se produzca un desplazamiento en valgo de la fractura "efecto cuña reverso".$^{16}$ Este tipo de desplazamiento secundario está descrito principalmente en las fracturas con trazo basicervical. Ante esta situación, las posibilidades son: introducir un gancho de hueso o "clamp colineal" y, de esta forma, generar una tracción lateral desde la cortical medial del cuello femoral. ${ }^{16}$ 


\section{POSICIONAMIENTO DEL ELEMENTO CEFÁLICO}

En términos de la posición del tornillo o la lámina en la cabeza femoral, existe bastante consenso en que la posición ideal en el perfil es el centro. ${ }^{6}$ Sin embargo, en la imagen de frente, al igual que nosotros, hay quienes defienden la ubicación centro ${ }^{6}$ y quienes, la ubicación inferior. ${ }^{17}$ En la práctica, un tornillo en posición inferior con buenos resultados clínicos, muchas veces, se asociaba a un índice punta-ápice $>25 \mathrm{~mm}$; por lo tanto, fue necesario describir un nuevo índice predictivo de falla que favorezca esta posición inferior del tornillo/lámina. El índice "Cal TAD”, descrito por Kuzyk y cols., en 2012, se diferencia del índice punta-ápice, descrito por Baumgaertner, ${ }^{6}$ solo en la imagen de frente. Utiliza una línea paralela al cuello femoral y adyacente al calcar, en lugar de correr por el centro del cuello. ${ }^{17}$

En cualquier situación, el concepto del índice punta-ápice es posicionar el elemento cefálico en la zona de mejor hueso para garantizar la mayor fijación posible y disminuir las posibilidades de "cut-out".

Una vez establecida la posición deseada en la cabeza, es posible que la introducción de la lámina o el tornillo provoque un desplazamiento rotatorio de la fractura. Esto es más probable en las fracturas basicervicales o en las fracturas conminutas. Una forma de evitar este desplazamiento es realizando una fijación temporal de la reducción lograda inicialmente con clavijas de $3,2 \mathrm{~mm}$ por fuera del sistema de guías del clavo. No obstante, si la fractura no fue estabilizada inicialmente y se produce la rotación, lo indicado es extraer el tornillo y volver a introducirlo luego de la estabilización controlando con el intensificador de imágenes. Si, cuando se llega con la punta del tornillo a la posición deseada, se observa algún defecto rotatorio, se deberá retirar el tornillo lo necesario para corregir la deformidad en el intensificador de imágenes.

Otra posibilidad es que, por la tracción necesaria para lograr la reducción o por la introducción del tornillo o lámina, se genere una distracción del foco de fractura. Si esto ocurre, luego de introducir la lámina o el tornillo, se deberá liberar la tracción sobre la extremidad y, por medio del sistema de compresión, completar la reducción de la fractura.

\section{CLAVO CORTO VS. CLAVO LARGO}

Como la inmensa mayoría de las fracturas intertrocantéricas ocurren en pacientes añosos con osteoporosis, es razonable pensar que un implante de mayor longitud protegerá al fémur de una segunda fractura. Por el contrario, Curtis y cols. comunicaron una incidencia más alta de fracturas alrededor del implante en la región metafisaria que en la región diafisaria. ${ }^{18}$ Sin embargo, los resultados de un metanálisis de 2019 muestran que el riesgo de fractura secundaria, falla de la osteosíntesis, seudoartrosis o infección fue similar entre los clavos largos y cortos. Solo fue significativo un tiempo quirúrgico más prolongado para colocar los clavos largos debido a la necesidad de fresar y de acerrojar a distal, a manos libres. ${ }^{19}$ Nuestra conducta de utilizar clavos cortos, como rutina, no solo se debe al menor tiempo quirúrgico, sino también a que, si se genera una fractura distal a este, el recambio de un clavo corto por uno más largo sería más simple que el tratamiento de una fractura supracondílea con una placa de fémur distal sobre el clavo.

\section{LÁMINA VS. TORNILLOS}

La adecuada fijación del implante a la cabeza femoral es un factor determinante para el éxito de la osteosíntesis. Algunos autores sostienen que son las fuerzas de rotación las que causan una pérdida de fijación del tornillo, el posterior colapso en varo de la cabeza femoral y finalmente el "cut-out". ${ }^{20}$ En estudios biomecánicos, se ha demostrado que las láminas resisten mejor las fuerzas de rotación, debido a su geometría y a su ingreso compactando radialmente en el hueso. ${ }^{21}$ Estudios clínicos han avalado los resultados de laboratorio. En un metanálisis de 2015, Shuang Li y cols. demostraron que el riesgo de "cut-out" es significativamente menor en el grupo de las láminas que en el de los tornillos. ${ }^{22}$ Por otro lado, en 2019, Ibrahim y cols. no hallaron diferencias estadísticamente significativas en el índice de falla entre el grupo de láminas y el de tornillos. Sin embargo, mencionan una diferencia en el patrón de falla entre ambos grupos: la migración axial y la penetración intrarticular fueron más frecuentes con la lámina. ${ }^{23}$

\section{BLOQUEO DISTAL}

La transferencia de carga en el fémur proximal luego de una osteosíntesis con un clavo de fémur proximal dependerá del patrón de fractura y la calidad de la reducción. En las fracturas inestables, todas las cargas serán transmitidas al fémur distal por el cerrojo de bloqueo del clavo hasta la consolidación o hasta que se rompa. Mientras que, en las fracturas estables, si la reducción es adecuada, el íntimo contacto cortical transmitirá directamente 
las cargas al fémur distal. Por ello, algunos autores han cuestionado la utilidad del bloqueo distal en las fracturas intertrocantéricas estables. ${ }^{24}$ En 2019, Yan y cols. llevaron a cabo un metanálisis sobre la necesidad o no de realizar el cerrojo distal del clavo en las fracturas estables y hallaron que el tiempo quirúrgico, el tiempo de exposición a la radioscopia eran más cortos, que había menos sangrado y dolor de muslo en el posoperatorio en el grupo de clavos sin acerrojado distal. Al mismo tiempo, los resultados funcionales de ambos grupos fueron similares. ${ }^{25}$ Como el tiempo de irradiación adicional es mínimo con el uso de las guías, nuestra conducta de rutina es realizar el bloqueo distal. Con clavos que ofrezcan la posibilidad de dos cerrojos distales, elegimos siempre el más proximal para disminuir la concentración de estrés sobre la punta del clavo.

\section{CONCLUSIONES}

A pesar del gran avance y el desarrollo de implantes en los últimos años, los principales factores determinantes del resultado final de la fijación de las fracturas intertrocantéricas siguen siendo la calidad de la reducción y el correcto posicionamiento del implante. Conocer los diferentes errores que se pueden cometer durante cada uno de los pasos de la técnica quirúrgica resulta indispensable para poder evitarlos.

Conflicto de intereses: Los autores no declaran conflictos de intereses.

\section{BIBLIOGRAFÍA}

1. Radaideh AM, Qudah HA. Functional and radiological results of proximal femoral nail antirotation (PFNA) osteosynthesis in the treatment of unstable pertrochanteric fractures. J Clin Med 2018;7(4):78. https://doi.org/10.3390/jcm7040078

2. Hardy DC, Descamps PY, Krallis P, Fabeck L, Smets P, Bertens CL, et al. Use of an intramedullary hip-screw compared with a compression hip-screw with a plate for intertrochanteric femoral fractures: a prospective, randomized study of one hundred patients. J Bone Joint Surg Am 1998;80(5):618-30. https://doi.org/10.2106/00004623-199805000-00002

3. Baumgaertner MR, Curtin SL, Lindskog DM, Keggi JM. The value of the tip-apex distance in predicting failure of fixation of peritrochanteric fractures of the hip. J Bone Joint Surg Am 1995;77(7):1058-64. https://doi.org/10.2106/00004623-199507000-00012

4. Bidolegui F, Vindver G, Di Stefano C. Manejo de las fracturas inestables del fémur proximal con el clavo PFN de la AO/ASIF. Evaluación de una serie prospectiva de 100 casos. Rev Asoc Argent Ortop Traumatol 2008;73(1):55-62. Disponible en: http://www.aaot.org.ar/revista/2008/n1_vol73/art09.pdf

5. Koval KJ, Oh CK, Egol KA. Does a traction-internal rotation radiograph help to better evaluate fractures of the proximal femur? Bull NYU Hosp Jt Dis 2008;66(2):102-6. PMID: 18537778

6. Lyon T, Koval KJ, Kummer F, Zuckerman JD. Pudendal nerve palsy induced by fracture table. Orthop Rev 1993; 22(5):521-5. PMID: 8316416

7. Rikli D, Goldhahn S, Blauth M, Mehta S, Cunningham M, Joeris A, PIP Study group. Optimizing intraoperative imaging during proximal femoral fracture fixation - a performance improvement program for surgeons. Injury 2018;49(2):339-44. https://doi.org/10.1016/j.injury.2017.11.024

8. Yong-Cheol Yoon, Chang-Wug Oh, Jae-Ang Sim, Jong-Keon Oh. Intraoperative assessment of reduction quality during nail fixation of intertrochanteric fractures. Injury 2020;51(2):400-6. https://doi.org/10.1016/j.injury.2019.10.087

9. Ottolenghi CE, Japas LM. Lateral fractures of the femur neck: the extradigital type. Rev Chir Orthop Reparatrice Appar Mot 1964;50:389-98. PMID: 14186381 
10. Young Soo Chun, Hyunsup Oh, Yoon Je Cho, Kee Hyung Rhyu. Technique and early results of percutaneous reduction of sagittally unstable intertrochateric fractures. Clin Orthop Surg 2011;3(3):217-24. https://doi.org/10.4055/cios.2011.3.3.217

11. Yong-Cheol Yoon, Ashutosh Jha, Chang-Wug Oh, Senthil Kumar Durai, Young-Woo Kim, Jong-Hoon Kim, et al. The pointed clamp reduction technique for spiral subtrochanteric fractures: A technical note. Injury 2014;45(6):1000-5. https://doi.org/10.1016/j.injury.2014.01.007

12. Carr JB. The anterior and medial reduction of intertrochanteric fractures: A simple method to obtain a stable reduction. J Orthop Trauma 2007;21:485-9. https://doi.org/10.1097/BOT.0b013e31804797cf

13. Ostrum RF, Marcantonio A, Marburger R. A critical analysis of the eccentric starting point for trochanteric intramedullary femoral nailing. J Orthop Trauma 2005;19:681-6. https://doi/org/10.1097/01.bot.0000184145.75201.1b

14. O’Malley MJ, Kang KK, Azer E, Siska PA, Farrell DJ, Tarkin IS. Wedge effect following intramedullary hip screw fixation of intertrochanteric proximal femur fracture. Arch Orthop Trauma Surg 2015;135(10):1343-7. https://doi.org/10.1007/s00402-015-2280-0

15. Hak DJ, Bilat C. Avoiding varus malreduction during cephalomedullary nailing of intertrochanteric hip fractures. Arch Orthop Trauma Surg 2011;131:709-10. https://doi.org/10.1007/s00402-010-1182-4

16. Yu Zhang, Jun Hu, Xiang Li, Xiaodong Qin. Reverse wedge effect following intramedullary nailing of a basicervical trochanteric fracture variant combined with a mechanically compromised greater trochanter. $B M C$ Musculoskelet Disord 2020;21(1):195. https://doi.org/10.1186/s12891-020-03212-6

17. Kuzyk PR, Zdero R, Shah S, Olsen M, Waddell JP, Schemitsch EH. Femoral head lag screw position for cephalomedullary nails: a biomechanical analysis. J Orthop Trauma 2012;26:414-21. https://doi.org/10.1097/BOT.0b013e318229acca

18. Curtis R, Goldhahn J, Schwyn R, Regazzoni P, Suhm N. Fixation principles in metaphyseal bone-a patent based review. Osteoporos Int 2005;16(Suppl 2):S54-S64. https://doi.org/10.1007/ s00198-004-1763-6

19. Pernille Bovbjerg, Lonnie Froberg, Hagen Schmal. Short versus long intramedullary nails for treatment of intertrochanteric femur fractures (AO 31-A1 and AO 31-A2): a systematic review. Eur J Orthop Surg Traumatol 2019;29(8):1823-31. https://doi.org/10.1007/s00590-019-02495-3

20. Nobuaki Chinzei, Takafumi Hiranaka, Takahiro Niikura, Mitsuo Tsuji, Ryosuke Kuroda, Minoru Doita, et al. Comparison of the sliding and femoral head rotation among three different femoral head fixation devices for trochanteric fractures. Clin Orthop Surg 2015;7(3):291-7. https://doi.org/10.4055/cios.2015.7.3.291

21. Sommers MB, Roth C, Hall H, Kam BCC, Ehmke LW, Krieg JC, et al. A laboratory model to evaluate cutout resistance of implants for pertrochanteric fracture fixation. J Orthop Trauma 2004;18:361-8. https.//doi.org/10.1097/00005131-200407000-00006

22. Shuang Li, Shi-Min Chang, Wen-Xin Niu, Hui Ma. Comparison of tip apex distance and cut-out complications between helical blades and lag screws in intertrochanteric fractures among the elderly: a meta-analysis. J Orthop Sci 2015;20:1062-9. https://doi.org/10.1007/s00776-015-0770-0

23. Ibrahim I, Appleton PT, Wixted JJ, DeAngelis JP, Rodriguez EK. Implant cut-out following cephalomedullary nailing of intertrochanteric femur fractures: Are helical blades to blame? Injury 2019;50(4):926-30. https://doi.org/10.1016/j.injury.2019.02.015

24. Caiaffa V, Vicenti G, Mori C, Panella A, Conserva V, Conserva V, et al. Is distal locking with short intramedullary nails necessary in stable pertrochanteric fractures? A prospective, multicentre, randomised study. Injury 2016;47(Supp4):S98-S106. https//doi.org/10.1016/j.injury.2016.07.038

25. Wen-Shan Yan, Wei-Li Cao, Ming Sun, Deng-Yue Ma, Peng Zhang. Distal locked or unlocked nailing for stable intertrochanteric fractures? A meta-analysis. ANZ J Surg 2020;90(1-2):27-33. https://doi.org/10.1111/ans. 15232 